\title{
CLOSURE OF BERGMAN AND DIRICHLET SPACES IN THE BLOCH NORM
}

\author{
Bin Liu and Jouni Rättyä \\ University of Eastern Finland, Department of Physics and Mathematics \\ P.O. Box 111, 80101 Joensuu, Finland; binl@uef.fi \\ University of Eastern Finland, Department of Physics and Mathematics \\ P.O. Box 111, 80101 Joensuu, Finland; jouni.rattya@uef.fi
}

\begin{abstract}
The closure of the weighted Bergman and Dirichlet spaces in the Bloch norm is described in terms of a kind of Lusin area function and natural level sets. In certain special cases simpler characterizations are also offered. The results are applied in the study of interpolating Blaschke products.
\end{abstract}

\section{Introduction and main results}

Let $\mathcal{H}(\mathbf{D})$ denote the space of analytic functions in the unit disc $\mathbf{D}=\{z \in$ $\mathbf{C}:|z|<1\}$. For a nonnegative function $\omega \in L^{1}([0,1))$, the extension to $\mathbf{D}$, defined by $\omega(z)=\omega(|z|)$ for all $z \in \mathbf{D}$, is called a radial weight. For $0<p<\infty, n \in \mathbf{N} \cup\{0\}$ and such an $\omega$, the space $A_{\omega, n}^{p}$ consists of $f \in \mathcal{H}(\mathbf{D})$ such that

$$
\|f\|_{A_{\omega, n}^{p}}^{p}=\int_{\mathbf{D}}\left|f^{(n)}(z)\right|^{p} \omega(z) d A(z)+\sum_{j=0}^{n-1}\left|f^{(j)}(0)\right|^{p}<\infty,
$$

where $d A(z)=\frac{d x d y}{\pi}$ is the normalized Lebesgue area measure on $\mathbf{D}$. In this definition we understand that the sum does not exist if $n=0$. Throughout this paper $\omega$ satisfies $\widehat{\omega}(z)=\int_{|z|}^{1} \omega(s) d s>0$ for all $z \in \mathbf{D}$, for otherwise $A_{\omega, n}^{p}=\mathcal{H}(\mathbf{D})$. We write $A_{\omega}^{p}=A_{\omega, 0}^{p}$ and $\mathcal{D}_{\omega}^{p}=A_{\omega, 1}^{p}$ for the weighted Bergman and Dirichlet spaces, respectively. As usual, $A_{\alpha}^{p}$ and $\mathcal{D}_{\alpha}^{p}$ denote the classical weighted Bergman and Dirichlet spaces induced by the standard radial weight $\omega(z)=\left(1-|z|^{2}\right)^{\alpha}$, where $-1<\alpha<\infty$. For $f \in \mathcal{H}(\mathbf{D})$ and $0<r<1$, set

$$
M_{p}(r, f)=\left(\frac{1}{2 \pi} \int_{0}^{2 \pi}\left|f\left(r e^{i t}\right)\right|^{p} d t\right)^{\frac{1}{p}}, \quad 0<p<\infty,
$$

and $M_{\infty}(r, f)=\max _{|z|=r}|f(z)|$. For $0<p \leq \infty$, the Hardy space $H^{p}$ consists of $f \in \mathcal{H}(\mathbf{D})$ such that $\|f\|_{H^{p}}=\sup _{0<r<1} M_{p}(r, f)<\infty$. The Bloch space $\mathcal{B}$ consists of $f \in \mathcal{H}(\mathbf{D})$ satisfying

$$
\|f\|_{\mathcal{B}}=\sup _{z \in \mathbf{D}}\left|f^{\prime}(z)\right|\left(1-|z|^{2}\right)+|f(0)|<\infty .
$$

Finally, a function $f \in H^{1}$ belongs to BMOA if it has bounded mean oscillation on the boundary $\mathbf{T}$ of $\mathbf{D}$, i.e. if it is the Poisson extension of a function in BMO of $\mathbf{T}$.

https://doi.org/10.5186/aasfm.2020.4533

2010 Mathematics Subject Classification: Primary 30H30; Secondary 46E15.

Key words: Bergman space, Blaschke product, Bloch space, closure in Bloch space, Dirichlet space, doubling weight, Lusin area function.

The first author is supported by China Scholarship Council, and the second by the Academy of Finland 286877. 
Garnett and Jones used the John-Nirenberg inequality [23] to characterize the closure of $L^{\infty}$ in BMO, and the closure of $H^{\infty}$ in BMOA [14]. Ghatage and Zheng in turn described the closure of BMOA in the Bloch norm [15], and they attributed the proof to Jones. A characterization of the closure of $H^{p} \cap \mathcal{B}$ in $\mathcal{B}$ was given by Galán and Nicolau when $1<p<\infty$ [27], and by Galanopoulos, Galán and Pau for $0<p \leq 1$ [12]. Very recently, Galanopoulos and Girela described the closure of $\mathcal{D}_{\alpha}^{p} \cap \mathcal{B}$ in $\mathcal{B}$ with $1 \leq p<\infty[11]$.

Our main purpose in this paper is to describe the closure of $A_{\omega, n}^{p} \cap \mathcal{B}$ in the Bloch space $\mathcal{B}$ for all $0<p<\infty$ and $n \in \mathbf{N} \cup\{0\}$, with relatively weak regularity hypotheses on the inducing radial weight $\omega$. In particular, our results cover the cases of the weighted Bergman and Dirichlet spaces for all $0<p<\infty$, and thus complete in part the recent study by Galanopoulos and Girela. Of course the results are of interest only if the space in question does not contain the whole Bloch space which is the case, for example, if $\omega$ tends to infinity sufficiently fast when approaching the boundary.

To state the results, some more definitions are needed. A radial weight $\omega$ belongs to the class $\widehat{\mathcal{D}}$ if the tail integral $\widehat{\omega}$ satisfies the doubling property $\widehat{\omega}(r) \leq C \widehat{\omega}\left(\frac{1+r}{2}\right)$ for some constant $C=C(\omega) \geq 1$ and for all $0 \leq r<1$. Weights in $\widehat{\mathcal{D}}$ can be characterized in terms of the moments $\omega_{x}=\int_{0}^{1} r^{x} \omega(r) d r$ of the weight; $\omega \in \widehat{\mathcal{D}}$ if and only if there exists a constant $C=C(\omega)>0$ such that $\omega_{x} \leq C \omega_{2 x}$ for all $x \geq 1$. This is an immediate consequence of [29, Lemma 2.1]. Further, if there exist $K=K(\omega)>1$ and $C=C(\omega)>1$ such that $\widehat{\omega}(r) \geq C \widehat{\omega}\left(1-\frac{1-r}{K}\right)$ for all $0 \leq r<1$, then we write $\omega \in \breve{\mathcal{D}}$. We also denote $\mathcal{D}=\widehat{\mathcal{D}} \cap \breve{\mathcal{D}}$ for short. Furthermore, if there exist $C=C(\omega)>1$ and $K=K(\omega)>1$ such that $\omega_{x} \geq C \omega_{K x}$ for all $x \geq 1$, we write $\omega \in \mathcal{M}$. It is known that $\mathcal{D}=\widehat{\mathcal{D}} \cap \mathcal{M}$ by [33, Theorem 3] and $\breve{\mathcal{D}} \subsetneq \mathcal{M}$ by [33, Proposition 13]. For basic properties of weights in these classes and more, see $[29,33,30,31,32]$ and the references therein.

For $\zeta=r e^{i \theta} \in \overline{\mathbf{D}} \backslash\{0\}$, a non-tangential approach region with vertex at $\zeta$ is defined by $\Gamma(\zeta)=\left\{z \in \mathbf{D}:|\theta-\arg z|<\frac{1}{2}\left(1-\frac{|z|}{r}\right)\right\}$. For $f \in \mathcal{H}(\mathbf{D}), m \in \mathbf{N}$ and $\varepsilon>0$, denote $\Omega_{m, \varepsilon}(f)=\left\{z \in \mathbf{D}:\left|f^{(m)}(z)\right|\left(1-|z|^{2}\right)^{m} \geq \varepsilon\right\}$. For a subspace $X$ of the Bloch space, let $\mathcal{C}_{\mathcal{B}}(X)$ denote the closure of the space $X$ in the Bloch norm.

The first main result of this study reads as follows.

Theorem 1. Let $0<p<\infty, n \in \mathbf{N} \cup\{0\}, \omega \in \widehat{\mathcal{D}}$ and $f \in \mathcal{B}$. Then $f \in$ $\mathcal{C}_{\mathcal{B}}\left(A_{\omega, n}^{p} \cap \mathcal{B}\right)$ if and only if

$$
\int_{\mathbf{D}}\left(\int_{\Gamma(\zeta) \cap \Omega_{n+1, \varepsilon}(f)} \frac{d A(z)}{\left(1-|z|^{2}\right)^{2(n+1)}}\right)^{\frac{p}{2}} \omega(\zeta) d A(\zeta)<\infty
$$

for all $\varepsilon>0$.

By putting [27, Theorem 1] and [12, Theorem 1] together, we see that $f \in \mathcal{B}$ belongs to $\mathcal{C}_{\mathcal{B}}\left(H^{p} \cap \mathcal{B}\right)$ if and only if

$$
\int_{\mathbf{T}}\left(\int_{\Gamma(\zeta) \cap \Omega_{1, \varepsilon}(f)} \frac{d A(z)}{\left(1-|z|^{2}\right)^{2}}\right)^{\frac{p}{2}}|d \zeta|<\infty
$$

for all $\varepsilon>0$. This is the natural limit case of Theorem 1 with $n=0$. 
If $p=2$ in Theorem 1, then Fubini's theorem and the fact that the integral over the tent $T(z)=\{\zeta \in \mathbf{D}: z \in \Gamma(\zeta)\}$ with respect to $\omega d A$ is comparable to $\widehat{\omega}(z)(1-|z|)$ for all $z \in \mathbf{D}$ by [29, Lemma 2.1] if $\omega \in \widehat{\mathcal{D}}$, yields the following result.

Corollary 2. Let $n \in \mathbf{N} \cup\{0\}, \omega \in \widehat{\mathcal{D}}$ and $f \in \mathcal{B}$. Then $f \in \mathcal{C}_{\mathcal{B}}\left(A_{\omega, n}^{2} \cap \mathcal{B}\right)$ if and only if

for all $\varepsilon>0$.

$$
\int_{\Omega_{n+1, \varepsilon}(f)} \frac{\widehat{\omega}(z)}{\left(1-|z|^{2}\right)^{2 n+1}} d A(z)<\infty
$$

In comparison to existing literature an interesting phenomenon occurs here. Namely, if we choose $n=1$, then Corollary 2 implies $f \in \mathcal{C}_{\mathcal{B}}\left(\mathcal{D}_{\omega}^{2} \cap \mathcal{B}\right)$ if and only if

$$
\int_{\Omega_{2, \varepsilon}(f)} \frac{\widehat{\omega}(z)}{\left(1-|z|^{2}\right)^{3}} d A(z)<\infty
$$

for all $\varepsilon>0$. But, by [11, Theorem 2], for each $1 \leq p<\infty$ and $-1<\alpha<\infty$ satisfying $p-2<\alpha \leq p-1$, we have $f \in \mathcal{C}_{\mathcal{B}}\left(\mathcal{D}_{\alpha}^{p} \cap \mathcal{B}\right)$ if and only if

$$
\int_{\Omega_{1, \varepsilon}(f)} \frac{d A(z)}{\left(1-|z|^{2}\right)^{p-\alpha}}<\infty
$$

for all $\varepsilon>0$. Now the integrand in $(1.1)$ for $\omega(z)=\left(1-|z|^{2}\right)^{\alpha}$ is comparable to $\left(1-|z|^{2}\right)^{\alpha-2}$, which is the integrand in (1.2) for $p=2$. However, the level sets are different: in (1.1) we integrate over $\Omega_{2, \varepsilon}(f)$ while (1.2) involves $\Omega_{1, \varepsilon}(f)$. The question then arises of when $\mathcal{C}_{\mathcal{B}}\left(A_{\omega, n}^{p} \cap \mathcal{B}\right)$ can be characterized in terms of a simpler condition such as that of Corollary 2 but involving the level set $\Omega_{n, \varepsilon}(f)$. The following result is a generalization of [11, Theorem 2] for doubling weights, and answers this question in the affirmative in certain cases.

Theorem 3. Let $1 \leq p<\infty, n \in \mathbf{N}$ and $\omega \in \mathcal{D}$ be such that

$$
\int_{0}^{r} \frac{\omega(t)}{(1-t)^{n p+1}} d t \lesssim \frac{\widehat{\omega}(r)}{(1-r)^{n p+1}}, \quad 0 \leq r<1
$$

and

$$
\int_{r}^{1} \frac{\omega(t)}{(1-t)^{n(p-1)}} d t \lesssim \frac{\widehat{\omega}(r)}{(1-r)^{n(p-1)}}, \quad 0 \leq r<1 .
$$

Then $f \in \mathcal{B}$ satisfies $f \in \mathcal{C}_{\mathcal{B}}\left(A_{\omega, n}^{p} \cap \mathcal{B}\right)$ if and only if

$$
\int_{\Omega_{n, \varepsilon}(f)} \frac{\widehat{\omega}(z)}{\left(1-|z|^{2}\right)^{n p+1}} d A(z)<\infty
$$

for all $\varepsilon>0$.

A bounded analytic function is an inner function if it has unimodular radial limits almost everywhere on the boundary $\mathbf{T}$. The question of when the derivative of an inner function belongs to the Hardy or the Bergman spaces has been a subject of research since 1970's. Membership of the derivative in the Hardy space $H^{p}$ and its Banach envelope $B^{p}$, with $0<p<1$, was studied in $[1,4,3,6,22,35]$. Derivatives of inner functions in the weighted Bergman space $A_{\alpha}^{p}$ has been studied in $[2,21,24]$, see $[10,16,17,18,19,20,25,37,36]$ for recent developments. See also the monographs [8] and [26]. Many known results concerning derivatives of inner functions in the classical weighted Bergman space $A_{\alpha}^{p}$ were recently generalized in [5] to the setting of $A_{\omega}^{p}$ induced by a normal weight $\omega$ in the sense of Shields and Williams [38]. Further generalizations to the case of doubling weights can be found in [34]. 
For a given sequence $\left\{z_{n}\right\}$ in $\mathbf{D}$ for which $\sum_{n}\left(1-\left|z_{n}\right|\right)$ converges, the Blaschke product associated with the sequence $\left\{z_{n}\right\}$ is defined as

$$
B(z)=\prod_{n} \frac{\left|z_{n}\right|}{z_{n}} \frac{z_{n}-z}{1-\bar{z}_{n} z}
$$

Recall that $\left\{z_{n}\right\}_{n=1}^{\infty}$ is uniformly separated, if there exists a constant $\delta>0$ such that

$$
\inf _{n \in \mathbf{N}} \prod_{k \neq n}\left|\frac{z_{k}-z_{n}}{1-\bar{z}_{k} z_{n}}\right|=\delta
$$

A Blaschke product is interpolating if its zero sequence is uniformly separated. Our last main result is the following characterization of such Blaschke products in $\mathcal{C}_{\mathcal{B}}\left(\mathcal{D}_{\omega}^{p} \cap\right.$ $\mathcal{B})$.

Theorem 4. Let $B$ be an interpolating Blaschke product, and let $1<p<\infty$ and $\omega \in \mathcal{D}$ such that

$$
\int_{0}^{r} \frac{\omega(t)}{(1-t)^{p}} d t \lesssim \frac{\widehat{\omega}(r)}{(1-r)^{p}}, \quad 0 \leq r<1,
$$

and

$$
\int_{r}^{1} \frac{\omega(t)}{(1-t)^{p-1}} d t \lesssim \frac{\widehat{\omega}(r)}{(1-r)^{p-1}}, \quad 0 \leq r<1
$$

Then the following statements are equivalent:

(i) $B \in \mathcal{C}_{\mathcal{B}}\left(\mathcal{D}_{\omega}^{p} \cap \mathcal{B}\right)$;

(ii) $B \in \mathcal{D}_{\omega}^{p}$;

(iii) $\sum_{n} \frac{\widehat{\omega}\left(z_{n}\right)}{\left(1-\left|z_{n}\right|\right)^{p-1}}<\infty$.

It is known that, under the hypotheses of Theorem 4 on $p$ and $\omega$, the Blaschke product $B$ associated with a finite union of separated sequences belongs to $\mathcal{D}_{\omega}^{p}$ if and only if the sum in (iii) converges [34, Theorem 1]. Therefore our contribution consists of showing that (i) and (iii) are equivalent. This equivalence is the counterpart of [11, Theorem 7] for weighted Dirichlet spaces induced by doubling weights.

The rest of the paper is organized as follows. In the next section we establish sets of necessary conditions for a function to belong to $\mathcal{C}_{\mathcal{B}}\left(A_{\omega, n}^{p} \cap \mathcal{B}\right)$. Sufficient conditions are considered in Section 3. The results on closures stated in the introduction are immediate consequences of these necessary and sufficient conditions. For convenience of the reader, this is detailed in Section 4 , where also a proof of Theorem 4 is given.

Before proving the results, a word about the notation used. The letter $C=C(\cdot)$ will denote an absolute constant whose value depends on the parameters indicated in the parenthesis, and may change from one occurrence to another. We will use the notation $a \lesssim b$ if there exists a constant $C=C(\cdot)>0$ such that $a \leq C b$ for all $a$ and $b$ under consideration, and $a \gtrsim b$ is understood in an analogous manner. In particular, if $a \lesssim b$ and $a \gtrsim b$, then we write $a \asymp b$ and say that $a$ and $b$ are comparable. 


\section{Necessary conditions}

It is known [40, Theorem 5.4] that for each $k \in \mathbf{N}$ we have

$$
\|f\|_{\mathcal{B}} \asymp \sup _{z \in \mathbf{D}}\left|f^{(k)}(z)\right|\left(1-|z|^{2}\right)^{k}+\sum_{j=0}^{k-1}\left|f^{(j)}(0)\right|=\|f\|_{\mathcal{B}, k}, \quad f \in \mathcal{H}(\mathbf{D}) .
$$

Proposition 5. Let $0<p<\infty, n \in \mathbf{N}, 0<\varepsilon<\infty$ and $\omega$ a radial weight, and let $f \in \mathcal{C}_{\mathcal{B}}\left(A_{\omega, n}^{p} \cap \mathcal{B}\right)$. Then the following statements hold:

(1) $\int_{\Omega_{n, \varepsilon}(f)} \frac{\omega(z)}{\left(1-|z|^{2}\right)^{n p}} d A(z)<\infty$;

(2) $\int_{\Omega_{m, \varepsilon}(f)} \frac{\omega(z)}{\left(1-|z|^{2}\right)^{n p}} d A(z)<\infty$ for all $m \geq n$, provided $\omega \in \widehat{\mathcal{D}}$;

(3) $\int_{\Omega_{m, \varepsilon}(f)} \frac{\widehat{\omega}(z)}{\left(1-|z|^{2}\right)^{n p+1}} d A(z)<\infty$ for all $m \geq n$, provided $\omega \in \mathcal{D}$.

Proof. Let $f \in \mathcal{C}_{\mathcal{B}}\left(A_{\omega, n}^{p} \cap \mathcal{B}\right)$ and $\varepsilon>0$. Then, by (2.1), for each $k \in \mathbf{N}$ there exists $g \in A_{\omega, n}^{p} \cap \mathcal{B}$ such that

$$
\sup _{z \in \mathbf{D}}\left|f^{(k)}(z)-g^{(k)}(z)\right|\left(1-|z|^{2}\right)^{k}<\frac{\varepsilon}{2} .
$$

By choosing $k=n$, it follows that

$$
\begin{aligned}
\infty & >\int_{\mathbf{D}}\left|g^{(n)}(z)\right|^{p} \omega(z) d A(z) \\
& \geq \int_{\Omega_{n, \varepsilon}(f)} \frac{\left|f^{(n)}(z)-f^{(n)}(z)+g^{(n)}(z)\right|^{p}\left(1-|z|^{2}\right)^{n p}}{\left(1-|z|^{2}\right)^{n p}} \omega(z) d A(z) \\
& \geq \int_{\Omega_{n, \varepsilon}(f)} \frac{\left(\left|f^{(n)}(z)\right|\left(1-|z|^{2}\right)^{n}-\left|f^{(n)}(z)-g^{(n)}(z)\right|\left(1-|z|^{2}\right)^{n}\right)^{p}}{\left(1-|z|^{2}\right)^{n p}} \omega(z) d A(z) \\
& >\left(\frac{\varepsilon}{2}\right)^{p} \int_{\Omega_{n, \varepsilon}(f)} \frac{\omega(z)}{\left(1-|z|^{2}\right)^{n p}} d A(z),
\end{aligned}
$$

and thus (1) is proved.

Next, observe that if $\omega \in \widehat{\mathcal{D}}$, then for each $m \geq n$ we have

$$
\int_{\mathbf{D}}\left|g^{(n)}(z)\right|^{p} \omega(z) d A(z) \gtrsim \int_{\mathbf{D}}\left|g^{(m)}(z)\right|^{p}\left(1-|z|^{2}\right)^{p(m-n)} \omega(z) d A(z), \quad g \in \mathcal{H}(\mathbf{D}),
$$

by [33, Theorem 6]. Pick $g \in A_{\omega, n}^{p} \cap \mathcal{B}$ such that (2.2) is satisfied for $k=m$. By following the proof above we deduce (2).

To see (3), observe first that if $\omega \in \mathcal{D}$, then $\omega_{[p(m-n)]} \in \mathcal{D}$ for $m \geq n$, where $\omega_{[\beta]}(z)=\omega(z)\left(1-|z|^{2}\right)^{\beta}$ for all $z \in \mathbf{D}$. Namely, $\mathcal{D}=\widehat{\mathcal{D}} \cap \breve{\mathcal{D}}=\widehat{\mathcal{D}} \cap \mathcal{M}$ by [33, Theorem 3], and [33, (1.2) and (1.3)] yield $\left(\omega_{[p(m-n)]}\right)_{x} \asymp x^{-p(m-n)} \omega_{x}$ for all $x \geq 1$ if $\omega \in \mathcal{D}$. Therefore $\left(\omega_{[p(m-n)]}\right)_{x} \asymp \omega_{x} x^{-p(m-n)} \lesssim \omega_{2 x}(2 x)^{-p(m-n)} \asymp\left(\omega_{[p(m-n)]}\right)_{2 x}$ by [29, Lemma 2.1], and thus $\omega_{[p(m-n)]} \in \widehat{\mathcal{D}}$ by the same lemma. The fact that $\omega_{[p(m-n)]} \in \widehat{\mathcal{D}}$ whenever $\omega \in \mathcal{D}$ can also be deduced by using $[34,(2.5)]$. Now that $\omega_{[p(m-n)]}$ trivially belongs to $\breve{\mathcal{D}}$ because $(1-r)^{p(m-n)}$ is decreasing in $r$, we deduce 
$\omega_{[p(m-n)]} \in \mathcal{D}$ whenever $\omega \in \mathcal{D}$. Therefore (2.3) and [31, Theorem 1] yield

$$
\begin{aligned}
\int_{\mathbf{D}}\left|f^{(n)}(z)\right|^{p} \omega(z) d A(z) & \gtrsim \int_{\mathbf{D}}\left|f^{(m)}(z)\right|^{p}\left(1-|z|^{2}\right)^{p(m-n)} \omega(z) d A(z) \\
& \gtrsim \int_{\mathbf{D}}\left|f^{(m)}(z)\right|^{p}\left(1-|z|^{2}\right)^{p(m-n)} \frac{\widehat{\omega}(z)}{1-|z|^{2}} d A(z)
\end{aligned}
$$

for all $f \in \mathcal{H}(\mathbf{D})$, provided $I(r) \lesssim I I(r)$ for all $0 \leq r<1$, where $I(r)=\int_{r}^{1} \frac{\widehat{\omega}(t)}{1-t}(1-t)^{p(m-n)} d t \quad$ and $\quad I I(r)=\int_{r}^{1} \omega(t)(1-t)^{p(m-n)} d t, \quad 0 \leq r<1$.

To prove this, note that the hypothesis $\omega \in \mathcal{D} \subset \breve{\mathcal{D}}$ yields

$$
\begin{aligned}
I(r) & =\int_{r}^{1-\frac{1-r}{K}} \frac{\widehat{\omega}(t)}{1-t}(1-t)^{p(m-n)} d t+\int_{1-\frac{1-r}{K}}^{1} \frac{\widehat{\omega}(t)}{1-t}(1-t)^{p(m-n)} d t \\
& =\int_{r}^{1-\frac{1-r}{K}} \frac{\widehat{\omega}(t)}{1-t}(1-t)^{p(m-n)} d t+\frac{1}{K^{p(m-n)}} \int_{r}^{1} \frac{\widehat{\omega}\left(1-\frac{1-s}{K}\right)}{1-s}(1-s)^{p(m-n)} d s \\
& \leq \int_{r}^{1-\frac{1-r}{K}} \frac{\widehat{\omega}(t)}{1-t}(1-t)^{p(m-n)} d t+\frac{I(r)}{C K^{p(m-n)}},
\end{aligned}
$$

which together with the hypothesis $\omega \in \mathcal{D}=\breve{\mathcal{D}} \cap \widehat{\mathcal{D}}$ and [29, Lemma 2.1] give

$$
\begin{aligned}
\left(1-\frac{1}{C K^{p(m-n)}}\right) I(r) & \leq \int_{r}^{1-\frac{1-r}{K}} \frac{\widehat{\omega}(t)}{1-t}(1-t)^{p(m-n)} d t \leq \widehat{\omega}(r)(1-r)^{p(m-n)} \log K \\
& \lesssim \widehat{\omega}\left(1-\frac{1-r}{K}\right)(1-r)^{p(m-n)} \frac{C-1}{C-1} \\
& \leq(1-r)^{p(m-n)} \frac{\widehat{\omega}(r)-\widehat{\omega}\left(1-\frac{1-r}{K}\right)}{C-1} \\
& \leq \frac{K^{p(m-n)}}{C-1} \int_{r}^{1-\frac{1-r}{K}} \omega(t)(1-t)^{p(m-n)} d t \lesssim I I(r) .
\end{aligned}
$$

Again the assertion follows as in the case (1).

Proposition 6. Let $0<p<\infty, n \in \mathbf{N} \cup\{0\}, 0<\varepsilon<\infty$ and $\omega$ a radial weight, and let $f \in \mathcal{C}_{\mathcal{B}}\left(A_{\omega, n}^{p} \cap \mathcal{B}\right)$. Then the following statements hold:

(1) $\int_{\mathbf{D}}\left(\int_{\Gamma(\zeta) \cap \Omega_{m, \varepsilon}(f)} \frac{\left(1-\left|\frac{z}{\zeta}\right|\right)^{2(m-n)-2}}{\left(1-|z|^{2}\right)^{2 m}} d A(z)\right)^{\frac{p}{2}} \omega(\zeta) d A(\zeta)<\infty$ for all $m \geq$ $n+1$;

(2) $\int_{\mathbf{D}}\left(\int_{\Gamma(\zeta) \cap \Omega_{k, \varepsilon}(f)} \frac{\left(1-\left|\frac{z}{\zeta}\right|\right)^{2(k-m)-2}}{\left(1-|z|^{2}\right)^{2 k}} d A(z)\right)^{\frac{p}{2}}(1-|\zeta|)^{(m-n) p} \omega(\zeta) d A(\zeta)<\infty$ for all $k-1 \geq m \geq n$, provided $\omega \in \widehat{\mathcal{D}}$;

(3) $\int_{\mathbf{D}}\left(\int_{\Gamma(\zeta) \cap \Omega_{k, \varepsilon}(f)} \frac{\left(1-\left|\frac{z}{\zeta}\right|\right)^{2(k-m)-2}}{\left(1-|z|^{2}\right)^{2 k}} d A(z)\right)^{\frac{p}{2}}(1-|\zeta|)^{(m-n) p-1} \widehat{\omega}(\zeta) d A(\zeta)<\infty$ for all $k-1 \geq m \geq n$, provided $\omega \in \mathcal{D}$. 
Proof. (1) Let $f \in \mathcal{C}_{\mathcal{B}}\left(A_{\omega, n}^{p} \cap \mathcal{B}\right)$ and $\varepsilon>0$. Then, by (2.1), for each $k \in \mathbf{N}$ there exists $g \in A_{\omega, n}^{p} \cap \mathcal{B}$ such that (2.2) holds. Moreover, for each $m \geq n+1$ and radial weight $\omega$ we have

$$
\begin{aligned}
\|h\|_{A_{\omega, n}^{p}}^{p} \asymp & \int_{\mathbf{D}}\left(\int_{\Gamma(\zeta)}\left|h^{(m)}(z)\right|^{2}\left(1-\left|\frac{z}{\zeta}\right|\right)^{2(m-n)-2} d A(z)\right)^{\frac{p}{2}} \omega(\zeta) d A(\zeta) \\
& +\sum_{j=n}^{m-1}\left|h^{(j)}(0)\right|^{p}
\end{aligned}
$$

for all $h \in \mathcal{H}(\mathbf{D})$, by [30, Theorem 4.2]. Pick $g \in A_{\omega, n}^{p} \cap \mathcal{B}$ such that (2.2) is satisfied for $k=m$, and then continue as in the proof of Proposition 5 with the aid of (2.5) to get

$$
\begin{aligned}
\infty & \|g\|_{A_{\omega, n}^{p}}^{p} \gtrsim \int_{\mathbf{D}}\left(\int_{\Gamma(\zeta)}\left|g^{(m)}(z)\right|^{2}\left(1-\left|\frac{z}{\zeta}\right|\right)^{2(m-n)-2} d A(z)\right)^{\frac{p}{2}} \omega(\zeta) d A(\zeta) \\
\geq & \int_{\mathbf{D}}\left(\int_{\Gamma(\zeta) \cap \Omega_{m, \varepsilon}(f)} \frac{\left|f^{(m)}(z)-f^{(m)}(z)+g^{(m)}(z)\right|^{2}\left(1-|z|^{2}\right)^{2 m}}{\left(1-\left|\frac{z}{\zeta}\right|\right)^{-2(m-n)+2}\left(1-|z|^{2}\right)^{2 m}} d A(z)\right)^{\frac{p}{2}} \omega(\zeta) d A(\zeta) \\
\geq & \int_{\mathbf{D}}\left(\int_{\Gamma(\zeta) \cap \Omega_{m, \varepsilon}(f)} \frac{\left(\left|f^{(m)}(z)\right|\left(1-|z|^{2}\right)^{m}-\left|f^{(m)}(z)-g^{(m)}(z)\right|\left(1-|z|^{2}\right)^{m}\right)^{2}}{\left(1-\left|\frac{z}{\zeta}\right|\right)^{-2(m-n)-2}\left(1-|z|^{2}\right)^{2 m}} d A(z)\right)^{\frac{p}{2}} \\
& \cdot \omega(\zeta) d A(\zeta) \\
\geq & \left(\frac{\varepsilon}{2}\right)^{p} \int_{\mathbf{D}}\left(\int_{\Gamma(\zeta) \cap \Omega_{m, \varepsilon}(f)} \frac{\left(1-\left|\frac{z}{\zeta}\right|\right)^{2(m-n)-2}}{\left(1-|z|^{2}\right)^{2 m}} d A(z)\right)^{\frac{p}{2}} \omega(\zeta) d A(\zeta),
\end{aligned}
$$

and (1) follows.

To prove (2), observe first that for all $k-1 \geq m \geq n$ we have

$$
\begin{aligned}
\|g\|_{A_{\omega, n}^{p}}^{p} & \gtrsim \int_{\mathbf{D}}\left|g^{(m)}(z)\right|^{p}\left(1-|z|^{2}\right)^{(m-n) p} \omega(z) d A(z) \\
& \asymp \int_{\mathbf{D}}\left(\int_{\Gamma(\zeta)}\left|g^{(k)}(z)\right|^{2}\left(1-\left|\frac{z}{\zeta}\right|\right)^{2(k-m)-2} d A(z)\right)^{\frac{p}{2}}\left(1-|\zeta|^{2}\right)^{(m-n) p} \omega(\zeta) d A(\zeta)
\end{aligned}
$$

by (2.3) and (2.5). By following the proof above we get the assertion. The last assertion (3) follows by using (2.4) and the arguments above.

\section{Sufficient conditions}

Proposition 7. Let $1 \leq p \leq \infty, n \in \mathbf{N}$ and $\omega$ a radial weight such that

$$
\begin{array}{cl}
\int_{0}^{r} \frac{\omega(t)}{(1-t)^{n p+1}} d t \lesssim \frac{\widehat{\omega}(r)}{(1-r)^{n p+1}}, & 0 \leq r<1, \\
\int_{r}^{1} \frac{\omega(t)}{(1-t)^{n(p-1)}} d t \lesssim \frac{\widehat{\omega}(r)}{(1-r)^{n(p-1)}}, \quad 0 \leq r<1 .
\end{array}
$$


If $f \in \mathcal{B}$ satisfies

$$
\int_{\Omega_{m, \varepsilon}(f)} \frac{\widehat{\omega}(z)}{\left(1-|z|^{2}\right)^{n p+1}} d A(z)<\infty
$$

for all $\varepsilon>0$ and for some $m \in \mathbf{N}$ such that $m \leq n$, then $f \in \mathcal{C}_{\mathcal{B}}\left(A_{\omega, n}^{p} \cap \mathcal{B}\right)$.

Proof. We may assume without loss of generality that $f(0)=f^{\prime}(0)=\cdots=$ $f^{(m-1)}(0)=0$. Since $f \in \mathcal{B}$ by the hypothesis, we may use [40, Proposition 4.27] to write $f=f_{1}+f_{2}$, where

$$
\begin{aligned}
& f_{1}(z)=\frac{1}{m !} \int_{\Omega_{m, \varepsilon}(f)} \frac{f^{(m)}(\zeta)\left(1-|\zeta|^{2}\right)^{m}}{(1-z \bar{\zeta})^{2} \bar{\zeta}^{m}} d A(\zeta), \quad z \in \mathbf{D}, \\
& f_{2}(z)=\frac{1}{m !} \int_{\mathbf{D} \backslash \Omega_{m, \varepsilon}(f)} \frac{f^{(m)}(\zeta)\left(1-|\zeta|^{2}\right)^{m}}{(1-z \bar{\zeta})^{2} \bar{\zeta}^{m}} d A(\zeta), \quad z \in \mathbf{D} .
\end{aligned}
$$

We have

$$
\begin{aligned}
\left(1-|z|^{2}\right)^{m}\left|f_{2}^{(m)}(z)\right| & \leq(m+1)\left(1-|z|^{2}\right)^{m} \int_{\mathbf{D} \backslash \Omega_{m, \varepsilon}(f)} \frac{\left|f^{(m)}(\zeta)\right|\left(1-|\zeta|^{2}\right)^{m}}{|1-z \bar{\zeta}|^{2+m}} d A(\zeta) \\
& \leq(m+1) \varepsilon\left(1-|z|^{2}\right)^{m} \int_{\mathbf{D} \backslash \Omega_{m, \varepsilon}(f)} \frac{d A(\zeta)}{|1-z \bar{\zeta}|^{2+m}} \\
& \leq(m+1) \varepsilon\left(1-|z|^{2}\right)^{m} \int_{\mathbf{D}} \frac{d A(\zeta)}{|1-z \bar{\zeta}|^{2+m}} \asymp \varepsilon, \quad z \in \mathbf{D},
\end{aligned}
$$

and hence $\left\|f-f_{1}\right\|_{\mathcal{B}, m}=\left\|f_{2}\right\|_{\mathcal{B}, m} \lesssim \varepsilon$. Since $f \in \mathcal{B}$ by hypothesis, we also have $f_{1} \in \mathcal{B}$. To complete the proof, it remains to show that $f_{1} \in A_{\omega, n}^{p}$. To see this, observe first that the hypothesis $p \geq 1$ and Fubini's theorem yield

$$
\begin{aligned}
& \int_{\mathbf{D}}\left|f_{1}^{(n)}(z)\right|^{p} \omega(z) d A(z) \leq\left\|f_{1}\right\|_{\mathcal{B}, n}^{p-1} \int_{\mathbf{D}}\left|f_{1}^{(n)}(z)\right| \frac{\omega(z)}{\left(1-|z|^{2}\right)^{n(p-1)}} d A(z) \\
& \lesssim\left\|f_{1}\right\|_{\mathcal{B}, n}^{p-1} \int_{\mathbf{D}} \frac{\omega(z)}{\left(1-|z|^{2}\right)^{n(p-1)}}\left(\int_{\Omega_{m, \varepsilon}(f)} \frac{\left|f^{(m)}(\zeta)\right|\left(1-|\zeta|^{2}\right)^{m}}{|1-z \bar{\zeta}|^{2+n}} d A(\zeta)\right) d A(z) \\
& \lesssim\left\|f_{1}\right\|_{\mathcal{B}, n}^{p-1}\|f\|_{\mathcal{B}, m} \int_{\Omega_{m, \varepsilon}(f)}\left(\int_{\mathbf{D}} \frac{\omega(z)}{\left(1-|z|^{2}\right)^{n(p-1)}|1-z \bar{\zeta}|^{2+n}} d A(z)\right) d A(\zeta),
\end{aligned}
$$

where

$$
\begin{aligned}
& \int_{\mathbf{D}} \frac{\omega(z)}{\left(1-|z|^{2}\right)^{n(p-1)}|1-z \bar{\zeta}|^{2+n}} d A(z) \lesssim\left(\int_{0}^{|\zeta|}+\int_{|\zeta|}^{1}\right) \frac{\omega(r)}{(1-r)^{n(p-1)}(1-|\zeta| r)^{1+n}} d r \\
& \leq \int_{0}^{\zeta \zeta \mid} \frac{\omega(r)}{(1-r)^{n p+1}} d r+\frac{1}{(1-|\zeta|)^{1+n}} \int_{|\zeta|}^{1} \frac{\omega(r)}{(1-r)^{n(p-1)}} d r \lesssim \frac{\widehat{\omega}(\zeta)}{(1-|\zeta|)^{n p+1}}, \quad \zeta \in \mathbf{D},
\end{aligned}
$$

by the hypotheses (3.1) and (3.2). Therefore

$$
\int_{\mathbf{D}}\left|f_{1}^{(n)}(z)\right|^{p} \omega(z) d A(z) \lesssim\left\|f_{1}\right\|_{\mathcal{B}, n}^{p-1}\|f\|_{\mathcal{B}, m} \int_{\Omega_{m, \varepsilon}(f)} \frac{\widehat{\omega}(z)}{(1-|z|)^{n p+1}} d A(z)<\infty,
$$

and thus $f_{1} \in A_{\omega, n}^{p}$.

To shorten the notation, for $f \in \mathcal{H}(\mathbf{D}), m \in \mathbf{N}$ and $\zeta \in \mathbf{D} \backslash\{0\}$, denote

$$
A_{m}(f)(\zeta)=\left(\int_{\Gamma(\zeta)}\left|f^{(m)}(z)\right|^{2}\left(1-\left|\frac{z}{\zeta}\right|\right)^{2 m-2} d A(z)\right)^{\frac{1}{2}}
$$


and

$$
\Phi_{m, \varepsilon}(f)(\zeta)=\left(\int_{\Gamma(\zeta) \cap \Omega_{m, \varepsilon}(f)} \frac{d A(z)}{\left(1-|z|^{2}\right)^{2 m}}\right)^{\frac{1}{2}}
$$

An application of [30, Theorem 4.2] shows that

$$
\|f\|_{A_{\omega, n}^{p}}^{p} \asymp\left\|A_{m}\left(f^{(n)}\right)\right\|_{L_{\omega}^{p}}^{p}+\sum_{j=0}^{n+m-1}\left|f^{(j)}(0)\right|^{p}, \quad f \in \mathcal{H}(\mathbf{D}) .
$$

Proposition 8. Let $0<p<\infty, n \in \mathbf{N} \cup\{0\}, \omega \in \widehat{\mathcal{D}}$ and $f \in \mathcal{B}$. If $\Phi_{n+1, \varepsilon}(f) \in$ $L_{\omega}^{p}$ for all $\varepsilon>0$, then $f \in \mathcal{C}_{\mathcal{B}}\left(A_{\omega, n}^{p} \cap \mathcal{B}\right)$.

Proof. Assume without loss of generality that $f(0)=f^{\prime}(0)=\cdots=f^{(n)}(0)=0$. Let $\gamma>0$ to be fixed later depending on $\omega$. Since $f \in \mathcal{B}$ by the hypothesis, we have

$$
f(z)=\frac{1}{(\gamma+2) \cdots(\gamma+n+1)} \int_{\mathbf{D}} \frac{f^{(n+1)}(\zeta)\left(1-|\zeta|^{2}\right)^{n+1+\gamma}}{\bar{\zeta}^{n+1}(1-z \bar{\zeta})^{2+\gamma}} d A(\zeta), \quad z \in \mathbf{D},
$$

by [40, Propostion 4.27]. Write $f=f_{1}+f_{2}$, where

$$
\begin{aligned}
& f_{1}(z)=\frac{1}{(\gamma+2) \cdots(\gamma+n+1)} \int_{\Omega_{n+1, \varepsilon}(f)} \frac{f^{(n+1)}(\zeta)\left(1-|\zeta|^{2}\right)^{n+1+\gamma}}{\bar{\zeta}^{n+1}(1-z \bar{\zeta})^{2+\gamma}} d A(\zeta), \quad z \in \mathbf{D}, \\
& f_{2}(z)=\frac{1}{(\gamma+2) \cdots(\gamma+n+1)} \int_{\mathbf{D} \backslash \Omega_{n+1, \varepsilon}(f)} \frac{f^{(n+1)}(\zeta)\left(1-|\zeta|^{2}\right)^{n+1+\gamma}}{\bar{\zeta}^{n+1}(1-z \bar{\zeta})^{2+\gamma}} d A(\zeta), \quad z \in \mathbf{D} .
\end{aligned}
$$

Clearly,

$$
\left|f_{2}^{(n+1)}(z)\right| \leq(n+2+\gamma) \varepsilon \int_{\mathbf{D}} \frac{\left(1-|\zeta|^{2}\right)^{\gamma}}{|1-\bar{\zeta} z|^{n+3+\gamma}} d A(z), \quad z \in \mathbf{D}
$$

and hence $\left\|f-f_{1}\right\|_{\mathcal{B}} \asymp\left\|f_{2}\right\|_{\mathcal{B}, n+1} \lesssim \varepsilon$ by (2.1) and the classical Forelli-Rudin estimates from [40, Lemma 3.10]. To complete the proof it remains to show that $f_{1} \in A_{\omega, n}^{p}$. This will be done by using the equivalent norm given by (3.5). First observe that

$$
\left|f_{1}^{(n+1)}(z)\right|^{2} \lesssim\|f\|_{\mathcal{B}, n+1}^{2}\left(\int_{\Omega_{n+1, \varepsilon}(f)} \frac{\left(1-|\zeta|^{2}\right)^{\gamma}}{|1-z \bar{\zeta}|^{n+3+\gamma}} d A(\zeta)\right)^{2}, \quad z \in \mathbf{D},
$$

where by the Cauchy-Schwarz inequality and the Forelli-Rudin estimates we have

$$
\begin{aligned}
& \left(\int_{\Omega_{n+1, \varepsilon}(f)} \frac{\left(1-|\zeta|^{2}\right)^{\gamma}}{|1-z \bar{\zeta}|^{3+n+\gamma}} d A(\zeta)\right)^{2}=\left(\int_{\Omega_{n+1, \varepsilon}(f)} \frac{\left(1-|\zeta|^{2}\right)^{\frac{\gamma}{2}}}{|1-z \bar{\zeta}|^{\frac{3+\gamma}{2}+n}} \frac{\left(1-|\zeta|^{2}\right)^{\frac{\gamma}{2}}}{|1-z \bar{\zeta}|^{\frac{3+\gamma}{2}}} d A(\zeta)\right)^{2} \\
& \leq \int_{\Omega_{n+1, \varepsilon}(f)} \frac{\left(1-|\zeta|^{2}\right)^{\gamma}}{|1-z \bar{\zeta}|^{3+2 n+\gamma}} d A(\zeta) \int_{\mathbf{D}} \frac{\left(1-|\zeta|^{2}\right)^{\gamma}}{|1-z \bar{\zeta}|^{3+\gamma}} d A(\zeta) \\
& \lesssim \int_{\Omega_{n+1, \varepsilon}(f)} \frac{\left(1-|\zeta|^{2}\right)^{\gamma}}{|1-z \bar{\zeta}|^{3+2 n+\gamma}} d A(\zeta)\left(1-|z|^{2}\right)^{-1}, \quad z \in \mathbf{D} .
\end{aligned}
$$

Therefore Fubini's theorem yields

$$
A_{1}\left(f_{1}^{(n)}\right)(\xi)^{2} \lesssim \int_{\Omega_{n+1, \varepsilon}(f)}\left(\int_{\Gamma(\xi)} \frac{d A(z)}{|1-z \bar{\zeta}|^{3+2 n+\gamma}\left(1-|z|^{2}\right)}\right)\left(1-|\zeta|^{2}\right)^{\gamma} d A(\zeta) .
$$


If $z \in \Gamma(\xi)$ where $\xi \in \mathbf{D} \backslash\{0\}$, then

and hence

$$
\frac{1}{2}\left(1-\left|\frac{z}{\xi}\right|\right) \geq|\arg \xi-\arg z| \asymp\left|\frac{\xi}{|\xi|}-\frac{z}{|z|}\right|=\left|1-\frac{\bar{\xi} z}{|\xi||z|}\right|
$$

$$
\begin{aligned}
1-|z| & \leq|1-\bar{\xi} z|=\left|1-\frac{\bar{\xi} z}{|\xi||z|}+\frac{\bar{\xi} z}{|\xi||z|}-\bar{\xi} z\right| \\
& \lesssim \frac{1}{|\xi|}(|\xi|-|z|)+(1-|\xi||z|) \lesssim(1-|z|), \quad|\xi| \geq \frac{1}{2} .
\end{aligned}
$$

It follows that $\left(1-|z|^{2}\right) \asymp|1-z \bar{\xi}|$ for all $z \in \Gamma(\xi)$ and $\xi \in \mathbf{D} \backslash\{0\}$. Therefore an application of [28, Lemma 2.5] with $r=t=3+2 n+\gamma$ and $s=2+2 n+\gamma$ yields

$$
\begin{aligned}
\int_{\Gamma(\xi)} \frac{d A(z)}{|1-z \bar{\zeta}|^{3+2 n+\gamma}\left(1-|z|^{2}\right)} & \lesssim \int_{\mathbf{D}} \frac{\left(1-|z|^{2}\right)^{2+2 n+\gamma}}{|1-z \bar{\zeta}|^{3+2 n+\gamma}|1-z \bar{\xi}|^{3+2 n+\gamma}} d A(z) \\
& \lesssim \frac{1}{|1-\zeta \bar{\xi}|^{2+2 n+\gamma}}
\end{aligned}
$$

for all $\zeta \in \mathbf{D}$ and $\xi \in \mathbf{D} \backslash\{0\}$. Hence

$$
\begin{aligned}
\left\|A_{1}\left(f_{1}^{(n)}\right)\right\|_{L_{\omega}^{p}}^{p} & \lesssim \int_{\mathbf{D}}\left(\int_{\Omega_{n+1, \varepsilon}(f)} \frac{\left(1-|\zeta|^{2}\right)^{\gamma}}{|1-\xi \bar{\zeta}|^{2(n+1)+\gamma}} d A(\zeta)\right)^{\frac{p}{2}} \omega(\xi) d A(\xi) \\
& =\int_{\mathbf{D}}\left(\int_{\mathbf{D}}\left(\frac{1-|\zeta|^{2}}{|1-\xi \bar{\zeta}|}\right)^{2(n+1)+\gamma} d \mu_{n, f}(\zeta)\right)^{\frac{p}{2}} \omega(\xi) d A(\xi),
\end{aligned}
$$

where

$$
d \mu_{n, f}(\zeta)=\frac{\chi_{\Omega_{n+1, \varepsilon}(f)}(\zeta) d A(\zeta)}{\left(1-|\zeta|^{2}\right)^{2(n+1)}}, \quad \zeta \in \mathbf{D}
$$

By choosing $\gamma=\gamma(\omega)>0$ sufficient large, [31, Lemma 4] implies

$$
\left\|A_{1}\left(f_{1}^{(n)}\right)\right\|_{L_{\omega}^{p}}^{p} \lesssim \int_{\mathbf{D}}\left(\int_{\Gamma(\xi) \cap \Omega_{n+1, \varepsilon}(f)} \frac{d A(\zeta)}{\left(1-|\zeta|^{2}\right)^{2(n+1)}}\right)^{\frac{p}{2}} \omega(\xi) d A(\xi)=\left\|\Phi_{n+1, \varepsilon}(f)\right\|_{L_{\omega}^{p}}^{p},
$$

and thus $f \in A_{\omega, n}^{p}$ by (3.5) and the hypothesis $\Phi_{n+1, \varepsilon}(f) \in L_{\omega}^{p}$.

\section{Proofs of main results}

Theorem 1 is an immediate consequence of Proposition 6(1), with $m=n+1$, and Proposition 8. Further, Theorem 3 is an immediate consequence of Propositions 5(3) and 7 with $m=n$. It remains to prove our last main result that concerns Blaschke products.

Proof of Theorem 4. As mentioned in the introduction, (ii) and (iii) are equivalent (even for Carleson-Newman Blaschke products) by [34, Theorem 1]. To complete the proof, we show that (i) and (iii) are equivalent. Assume first $B \in \mathcal{C}_{\mathcal{B}}\left(\mathcal{D}_{\omega}^{p} \cap \mathcal{B}\right)$. Then, by Theorem 3, we have

$$
\int_{\Omega_{1, \varepsilon}(B)} \frac{\widehat{\omega}(z)}{\left(1-|z|^{2}\right)^{p+1}} d A(z)<\infty
$$

for all $\varepsilon>0$. Further, since $B$ is interpolating, [19, Lemma 3.5] implies the existence of $\varepsilon>0$ and $\delta>0$ such that the pseudohyperbolic discs $\Delta\left(z_{n}, \delta\right)=\left\{z \in \mathbf{D}: \mid z_{n}-\right.$ 
$\left.z|/| 1-\overline{z_{n}} z \mid<\delta\right\}$ are pairwise disjoint and $\left|B^{\prime}(z)\right|(1-|z|) \geq \varepsilon$ for all $z \in \bigcup_{n} \Delta\left(z_{n}, \delta\right)$. Therefore $\bigcup_{n} \Delta\left(z_{n}, \delta\right) \subset \Omega_{1, \varepsilon}(B)$. An application of [29, Lemma 2.1] shows that $\widehat{\omega}(z) \asymp \widehat{\omega}\left(z_{n}\right)$ for all $z \in \delta\left(z_{n}, \delta\right)$ and each $n$, and hence

$$
\begin{aligned}
\infty & >\int_{\Omega_{1, \varepsilon}(B)} \frac{\widehat{\omega}(z)}{\left(1-|z|^{2}\right)^{p+1}} d A(z) \\
& \geq \sum_{n} \int_{\Delta\left(z_{n}, \delta\right)} \frac{\widehat{\omega}(z)}{\left(1-|z|^{2}\right)^{p+1}} d A(z) \asymp \sum_{n} \frac{\widehat{\omega}\left(z_{n}\right)}{\left(1-\left|z_{n}\right|\right)^{p-1}} .
\end{aligned}
$$

Thus (iii) is satisfied.

Conversely, assume (iii). For each $z \in \Omega_{1, \varepsilon}(B)$ we have

$$
\frac{\varepsilon}{1-|z|^{2}} \leq\left|B^{\prime}(z)\right| \leq \sum_{n} \frac{1-\left|z_{n}\right|^{2}}{\left|1-\overline{z_{n}} z\right|^{2}} .
$$

Hence

$$
\begin{aligned}
\int_{\Omega_{1, \varepsilon}(B)} \frac{\widehat{\omega}(z)}{\left(1-|z|^{2}\right)^{p+1}} d A(z) & \lesssim \sum_{n}\left(1-\left|z_{n}\right|\right) \int_{\mathbf{D}} \frac{\widehat{\omega}(z)}{(1-|z|)^{p}\left|1-\overline{z_{n}} z\right|^{2}} d A(z) \\
& \asymp \sum_{n}\left(1-\left|z_{n}\right|\right) \int_{0}^{1} \frac{\widehat{\omega}(r)}{(1-r)^{p}\left(1-\left|z_{n}\right| r\right)} d r \lesssim I_{1}+I_{2}
\end{aligned}
$$

where

$$
I_{1}=\sum_{n}\left(1-\left|z_{n}\right|\right) \int_{0}^{\left|z_{n}\right|} \frac{\widehat{\omega}(r)}{(1-r)^{p+1}} d r
$$

and

$$
I_{2}=\sum_{n} \int_{\left|z_{n}\right|}^{1} \frac{\widehat{\omega}(r)}{(1-r)^{p}} d r .
$$

Fubini's theorem, (1.3) and the hypothesis yield

$$
\begin{aligned}
I_{1} & =\sum_{n}\left(1-\left|z_{n}\right|\right)\left(\widehat{\omega}\left(z_{n}\right) \int_{0}^{\left|z_{n}\right|} \frac{d r}{(1-r)^{p+1}}+\int_{0}^{\left|z_{n}\right|}\left(\int_{r}^{\left|z_{n}\right|} \omega(s) d s\right) \frac{d r}{(1-r)^{p+1}}\right) \\
& \asymp \sum_{n} \frac{\widehat{\omega}\left(z_{n}\right)}{\left(1-\left|z_{n}\right|\right)^{p-1}}+\sum_{n}\left(1-\left|z_{n}\right|\right) \int_{0}^{\left|z_{n}\right|} \frac{\omega(s)}{(1-s)^{p}} d s \lesssim \sum_{n} \frac{\widehat{\omega}\left(z_{n}\right)}{\left(1-\left|z_{n}\right|\right)^{p-1}}<\infty .
\end{aligned}
$$

Similarly, Fubini's theorem, (1.4) and the hypothesis yield

$$
\begin{aligned}
I_{2} & =\sum_{n} \int_{\left|z_{n}\right|}^{1} \omega(s)\left(\int_{\left|z_{n}\right|}^{s} \frac{d r}{(1-r)^{p}}\right) d s \lesssim \sum_{n} \int_{\left|z_{n}\right|}^{1} \frac{\omega(s)}{(1-s)^{p-1}} d s \\
& \lesssim \sum_{n} \frac{\widehat{\omega}\left(z_{n}\right)}{\left(1-\left|z_{n}\right|\right)^{p-1}}<\infty .
\end{aligned}
$$

Thus $B \in \mathcal{C}_{\mathcal{B}}\left(\mathcal{D}_{\omega}^{p} \cap \mathcal{B}\right)$ by Theorem 3 . 


\section{References}

[1] Ahern, P.: The mean modulus and the derivative of an inner function. - Indiana Univ. Math. J. 28:2, 1979, 311-347.

[2] Ahern, P.: The Poisson integral of a singular measure. - Canad. J. Math. 35:4, 1983, 735-749.

[3] Ahern, P. R., and D. N. Clark: On inner functions with $H^{p}$ derivative. - Michigan Math. J. 21, 1974, 115-127.

[4] Ahern, P. R., and D. N. Clark: On inner functions with $B^{p}$ derivative. - Michigan Math. J. 23:2, 1976, 107-118.

[5] Aleman, A., and D. Vukotić: On Blaschke products with derivatives in Bergman spaces with normal weights. - J. Math. Anal. Appl. 361:2, 2010, 492-505.

[6] Cohn, W. S.: On the $H^{p}$ classes of derivatives of functions orthogonal to invariant subspaces. - Michigan Math. J. 30:2, 1983, 221-229.

[7] Cohn, W. S.: Radial limits and star invariant subspaces of bounded mean oscillation. - Amer. J. Math. 108:3, 1986, 719-749.

[8] Colwell, P.: Blaschke products: bounded analytic functions. - Univ. of Michigan Press, Ann Arbor/Michigan, 1985.

[9] Duren, P.: Theory of $H^{p}$ spaces. - Academic Press, New York-London, 1970.

[10] Fricain, E., and J. Mashreghi: Integral means of the derivatives of Blaschke products. Glasg. Math. J. 50:2, 2008, 233-249.

[11] Galanopoulos, P., and D. Girela: The closure of Dirichlet spaces in the Bloch space. Ann. Acad. Sci. Fenn. Math. 44, 2019, 91-101.

[12] Galanopoulos, P., N. Monreal Galán, and J. Pau: Closure of Hardy spaces in the Bloch space. - J. Math. Anal. Appl. 429:2, 2015, 1214-1221.

[13] Garnett, J.: Bounded analytic functions. Revised 1st edition. - Springer, New York, 2007.

[14] Garnett, J. B., and P. W. Jones: The distance in BMO to $L^{\infty}$. - Ann. of Math. (2) 108:2, $1978,373-393$.

[15] Ghatage, P. G., and D. C. Zheng: Analytic functions of bounded mean oscillation and the Bloch space. - Integral Equations Operator Theory 17:4, 1993, 501-515.

[16] Girela, D., C. González, and M. Jevtić: Inner functions in Lipschitz, Besov, and Sobolev spaces. - Abstr. Appl. Anal. 2011, Art. ID 626254, 1-26.

[17] Girela, D., and J. A. PelÁez: On the derivative of infinite Blaschke products. - Illinois J. Math. 48:1, 2004, 121-130.

[18] Girela, D., and J. A. Peláez: On the membership in Bergman spaces of the derivative of a Blaschke product with zeros in a Stolz domain. - Canad. Math. Bull. 49:3, 2006, 381-388.

[19] Girela, D., J. A. Peláez, and D. Vukotić: Integrability of the derivative of a Blaschke product. - Proc. Edinb. Math. Soc. (2) 50:3, 2007, 673-687.

[20] Girela, D., J. A. Peláez, and D. Vukotić: Uniformly discrete sequences in regions with tangential approach to the unit circle. - Complex Var. Elliptic Equ. 52:2-3, 2007, 161-173.

[21] Gluchoff, A.: On inner functions with derivative in Bergman spaces. - Illinois J. Math. 31 (1987), no. 3, 518-528.

[22] Gröhn, J., and A. Nicolau: Inner functions in certain Hardy-Sobolev spaces. - J. Funct. Anal. 272:6, 2017, 2463-2486.

[23] John, F., and L. Nirenberg: On functions of bounded mean oscillation. - Comm. Pure Appl. Math. 14, 1961, 415-426.

[24] KIM, H. O.: Derivatives of Blaschke products. - Pacific J. Math. 114:1, 1984, 175-190.

[25] KutBi, M. A.: Integral means for the first derivative of Blaschke products. - Kodai Math. J. 24:1, 2001, 86-97. 
[26] Mashreghi, J.: Derivatives of inner functions. - Fields Inst. Monogr. 31, Springer, New York, Fields Institute for Research in Mathematical Sciences, Toronto, ON, 2013.

[27] Monreal Galán, N., and A. Nicolau: The closure of the Hardy space in the Bloch norm. - Algebra i Analiz 22:1, 2010, 75-81.

[28] Ortega, J. M., and J. Fàbrega: Pointwise multiplies and corona type decomposition in BMOA. - Ann. Inst. Fourier (Grenoble) 46, 1996, 111-137.

[29] PelÁez, J. A.: Small weighted Bergman spaces. - Proceedings of the summer school in complex and harmonic analysis, and related topics, 2016.

[30] PelÁEz, J.A., and J. RÄTtYä: Weighted Bergman spaces induced by rapidly increasing weights. - Mem. Amer. Math. Soc. 227:1066, 2014.

[31] PelÁez, J. A., and J. RÄTtYä: Embedding theorems for Bergman spaces via harmonic analysis. - Math. Ann. 362:1-2, 2015, 205-239.

[32] PelÁez, J. A., and J. RÄtträ: Two weight inequality for Bergman projection. - J. Math. Pures Appl. 105, 2016, 102-130.

[33] Peláez, J. A., and J. RÄTtYä: Bergman projection induced by radial weight. - Preprint, https://arxiv.org/abs/1902.09837.

[34] Pérez-González, F., J. RÄttyä, and A. Reijonen: Derivatives of inner functions in Bergman spaces induced by doubling weights. - Ann. Acad. Sci. Fenn. Math. 42, 2017, 735-753.

[35] Protas, D.: Blaschke products with derivative in $H^{p}$ and $B^{p}$. - Michigan Math. J. 20, 1973, 393-396.

[36] Protas, D.: Mean growth of the derivative of a Blaschke product. - Kodai Math. J. 27:3, 2004, 354-359.

[37] Protas, D.: Blaschke products with derivative in function spaces. - Kodai Math. J. 34:1, 2011, 124-131.

[38] Shields, A. L., and D. L. Williams: Bounded projections, duality, and multipliers in spaces of analytic functions. - Trans. Amer. Math. Soc. 162, 1971, 287-302.

[39] ZHU, K.: Bloch type spaces of analytic functions. - Rocky Mountain J. Math. 23, 1993, 11431177.

[40] ZHU, K.: Operator theory in function spaces. Second edition. - Math. Surveys Monogr. 138, Amer. Math. Soc., Providence, Rhode Island, 2007.

Received 6 May $2019 \bullet$ Accepted 6 August 2019 